\title{
Magnitude and factors influencing unintended pregnancy among pregnant women attending antenatal care at Felege Hiwot referral hospital, Northwest Ethiopia: A cross-sectional study
}

\author{
Wubalem Gebreamlak ${ }^{1, ~ *, ~ A m a n u ~ A r a g a w ², ~ S e b l e w o n g e l e ~ L e m m a ~}{ }^{3}$, Wubante Demilew ${ }^{4}$ \\ ${ }^{1}$ Department of Midwifery, college of Health sciences, Bahir Dar Health Science College, Bahir Dar City \\ ${ }^{2}$ Department of Nursing, college of Health sciences, Bahir Dar Health Science College, Bahir Dar City \\ ${ }^{3}$ Department of Epidemiology and Biostatistics, Addis continental institute of public health, Addis Ababa \\ ${ }^{4}$ Department of pharmacy, college of Health sciences, Bahir Dar Health Science College, Bahir Dar City
}

\section{Email address:}

wubie2001@gmail.com (W. Gebreamlak), amanuaragaw@yahoo.com (A. Aragaw), sebeye2007@gmail.com (S. Lemma), wubanted@yahoo.com (W. Demilew)

\section{To cite this article:}

Wubalem Gebreamlak, Amanu Aragaw, Seblewongele Lemma, Wubante Demilew. Magnitude and Factors Influencing Unintended Pregnancy among Pregnant Women Attending Antenatal Care at Felege Hiwot Referral Hospital, Northwest Ethiopia: A Cross-Sectional Study. Science Journal of Public Health. Vol. 2, No. 4, 2014, pp. 261-269. doi: 10.11648/j.sjph.20140204.13

\begin{abstract}
Background: Unintended pregnancy is important public health concern both in the developing and developed world that increases maternal morbidity and mortality. The proportion of unintended pregnancies is increasing in Ethiopia; yet the determinants of unwanted and mistimed pregnancy has not been identified clearly. The objectives of this study were to determine magnitude and identify factors associated with unintended pregnancy among currently pregnant women visiting antenatal care clinic, Bahir Dar, Ethiopia. Methods: A cross sectional study was conducted on 454 women attending antenatal care clinic at Felege Hiwot Referral Hospital from June to July 2012. Simple random sampling technique was used to select study participants. Data were collected by trained data collectors using pretested structured questionnaires. Data were checked for completeness, consistency, coded and entered into EPI Info (version 3.5.2) and transferred to SPSS version 16 for analysis. Bivariate and multivariate logistic regression analysis was computed to test the strength of association and level of significance. P-value $<0.05$ was considered as statistical significant. Results: The magnitude of unintended pregnancy was $26.0 \%(13.7 \%$ mistimed and $12.3 \%$ were unwanted). Major reasons mentioned for failure to avoid unintended pregnancy were lack of knowledge, disapproval by husband, and method failure. The study revealed that illiterate women were three times more likely to experience unintended pregnancy compared to those women educated secondary and above (AOR=3.10, $\mathrm{CI}$ : 1.66- 5.78). Likewise, those women who had family size of 3-5 were twice more likely at risk to have unintended pregnancy compared to those who had family size of 1-2 (AOR=2.19; CI: 1.32- 3.61) and those women who had family size of greater than 5 were nine folds at risk to have unintended pregnancy compared to those who had family size of $1-2$ (AOR=8.90; CI: 4.37-18.13). Conclusion: The finding of this study showed that a considerable proportion of women had unintended pregnancy (26\%). The study showed that many factors were interwoven to affect the occurrence of the unintended pregnancy. Differences in educational status of women and family size, previous history of unintended pregnancy and male partner awareness on contraceptive utilization were found to be significantly significant with unintended pregnancy. Health promotion messages are needed to focus to improve the knowledge of women about contraceptives as a primary prevention of unplanned pregnancies.
\end{abstract}

Keywords: Magnitude, Factors, Unintended Pregnancy, Pregnant Women, Antenatal Care, Ethiopia

\section{Background}

Unintended pregnancies are pregnancies that are reported to have been either unwanted (i.e., they occurred when no children, or no more children, were desired) or mistimed (i.e., they occurred earlier than desired) [1]. Unintended pregnancy can result from not using contraceptives, contraceptive failure and less commonly, from rape [2]. 
Unintended pregnancy is a worldwide problem that affects women, their families and societies at large. It is an important public health concern in both the developing and developed world because of its association with adverse social and health outcomes, for both mothers and children. Unintended pregnancies particularly among women in developing countries are linked to elevated maternal morbidity and mortality $[1,2,3]$.

It has been estimated that every day, 1,600 women and more than 10,000 newborns die from preventable complications during pregnancy and childbirth in which a large proportion $(\sim 50 \%)$ of such pregnancies are unplanned and about $25 \%$ are definitely unwanted. Almost $99 \%$ of these maternal and $90 \%$ of neonatal deaths occur in the developing countries. The lifetime risk of dying of pregnancy-related conditions is 1 in 60 in developing countries. In Sub-Saharan Africa, maternal mortality occurs in 1 in 160 pregnancies [3, 4].

In low-income countries, because less availability and options low use of contraception continues to be the main factor influencing the prevalence of unintended pregnancy [1]. Low contraception use has been linked to poor access to reproductive health services, gender norms and sexual abuse or coercion [5].

Many women and couples in Ethiopia do not have the knowledge, tools or assistance needed to maintain their reproductive health and have the number of children they desire $[6,7]$.

According to report of the Ethiopian Demographic and Health Survey 2011, 25\% of currently married women had an unmet need for family planning; $9 \%$ of births were not wanted and $16 \%$ of births were mistimed [8].

Maternal mortality in Ethiopia is one of the highest in the world. Few surveys conducted on issues related to unwanted pregnancy suggested that unwanted pregnancy was among the main causes of maternal mortality in the country $[8,9,10]$.

It is a universally accepted fact that unintended pregnancy and births could have negative consequences for women, children, families and societies at large. It is one of the major factors for increased maternal mortality in Ethiopia, perhaps in Amhara region, Northwest Ethiopia as well. It is also imperative that a persistent study must be conducted to elucidate some of the predictors that determine unintended pregnancy in order to reduce maternal morbidity and mortality related to unintended pregnancy to assist in maternal mortality reduction program of the millennium development goal. Thus, this study is believed to establish an insight about current unintended pregnancy rate and its determinants that may herald stakeholders in designing strategies to reduce maternal mortality.

In Ethiopia, few studies were conducted but no study has been found in the target area. Therefore, this study was aimed to assess the magnitude of unintended pregnancy and associated factors so as to initiate intervention by the concerned authorities.

\section{Participants and Methods}

\subsection{Study Area}

The study was conducted in Felege Hiwot Referral Hospital, Bahir Dar City. Bahir Dar is the capital of the Amhara Regional state, Ethiopia. The city is located approximately $565 \mathrm{~km}$ Northwest of Addis Ababa, capital city of Ethiopia. The city is divided in to nine administrative kebeles, the smallest administrative units in Ethiopia. The number of females in reproductive age are expected to be 64,803 ( $24 \%$ of the total population).Total fertility rate (TFR) was estimated to be 2.6 children per woman of reproductive age, while the overall national figure was 4.8 children per woman of reproductive age group [7].

\subsection{Study Design and Period}

Institution based cross-sectional study was conducted from June to July 2012 at Felege Hiwot Referral Hospital, Bahir Dar City, Northwest Ethiopia.

\subsection{Study Population}

Pregnant women who were registered and attended ANC in Felege Hiwot Hospital during the study period were recruited in the study. Pregnant women that were registered and interviewed once but who came for the second or subsequent visits and seriously ill pregnant women were excluded from the study.

\subsection{Variables}

While unintended pregnancy was the dependent variable, Socio demographic characteristics such as age, duration in marriage, residence, educational status; knowledge and practice about contraceptive; spousal communication; exposure to mass media, and reproductive history were the covariates.

\subsection{Sample Size Determination and Sampling Procedures}

The sample size was determined based on the result of proportion of unintended pregnancy in Amhara Region obtained from EDHS 2011, which was 22.1\% [28]. The following assumptions were used: level of confidence $95 \%$, a $4 \%$ marginal error. Based on these assumptions and taking $10 \%$ non response rate, the sample size calculated was 454 using single proportion formula $\left(\mathrm{N}=(\mathrm{za} / 2)^{2} \mathrm{p}(1-\mathrm{p})\right.$ /d2).

Simple random sampling technique was used to select the study participants using ANC registration book that contained list of pregnant women who visited prenatal clinic as a sampling frame.

\subsection{Data Collection}

Structured questionnaires which comprised of two parts were the study instruments. The first part contained sociodemographic and economic factors and the second part was 
on reproductive history of pregnant women. The questionnaire was initially prepared in English and was translated in to Amharic (local language) and again retranslated back to English to check for any inconsistencies or distortions in the meaning of words and concepts. Face to face interview was the technique of data collection and female data collectors were recruited to get frank response of respondents by taking into consideration of women's preference in the community.

\subsection{Data Quality Assurance}

To assure data quality the instrument was retranslated to local language. The recruited data collectors and their supervisors were trained for one day. A questionnaire was pre-tested in Han health center (one of the health centers in the city administration) on 25 women and modifications were done accordingly. Close supervision was undertaken during data collection and every questionnaire was crosschecked daily by the supervisor and the principal investigator and Problems faced were discussed overnight.

\subsection{Data Management and Analysis}

All the collected data were coded, checked for completeness and consistency. The data were entered into EPI Info version 3.5.2 and transferred to SPSS version 16.0 statistical soft ware for its analysis.

Descriptive and bivariate analyses were computed to see the frequency distribution and to test whether there is association between dependent variable and selected independent variables respectively. Factors associated with unintended pregnancy at bivariate analysis were identified and the variables with p-value of 0.20 and less were fit to logistic model for multivariable analysis. P-value less than 0.05 at $95 \%$ confidence interval was considered as statistically significant.

\subsection{Ethical Consideration}

Ethical clearance was obtained from the ethics review committee of Addis continental institute of public health, (ACIPH) and letter of permission was obtained from the administrators of Felege Hiwot Referral Hospital. All the study participants were informed about the purpose of the study, their right to refuse and assurance of confidentiality. Oral informed verbal consent was obtained from every respondent. Strict confidentiality was also maintained through anonymous recording and coding of questionnaire.

\section{Results}

\subsection{Socio Demographic Characteristics}

Four hundred fifty four pregnant women in reproductive age group were participated and interviewed in the study. Majority of the participants, 360 (79.3\%), were urban residents. The age of respondents ranged from 15-48 years with the mean $( \pm \mathrm{SD})$ of $25.8( \pm 5.3)$ years, and half of respondents $230(50.7 \%)$ were in the age group of 25-34 years. About $250(55.1 \%)$ of the respondents had three or more family members and the median of family size was 3 members (inter quartile range of 2, 3, 4 family members for $25,50,75$ percentiles respectively).The majority of respondents 445 (98.0\%) were Amhara in Ethnicity and $402(88.5 \%)$ were Orthodox Christians by religion. Concerning duration in marriage, a little less than half, 212 $(46.7 \%)$ were lived together with their partners for $0-5$ years and only $31(6.8 \%)$ of them were lived together beyond two decades. Regarding the occupational status, majority of the study participants $(43.8 \%)$ were house wives and only $(5.1 \%)$ of the respondents were students. About 179(39.4) of them had monthly income less than 1000.00 Ethiopian birr (Table 1).

Regarding the educational status of the study participants (27.5\%) were illiterate (unable to read and write), and a quarter, $114(25.1 \%)$, of women had completed their higher education (figure 1).

Table 1. Socio demographic characteristic of pregnant women attending ANC clinic in Felege Hiwot Referral Hospital, Bahir Dar; July, 2012 $(n=454)$

\begin{tabular}{|c|c|c|}
\hline $\begin{array}{l}\text { Socio demographic } \\
\text { characteristics }\end{array}$ & Number & percent \\
\hline \multicolumn{3}{|l|}{ Address } \\
\hline Urban & 360 & 79.3 \\
\hline Rural & 94 & 20.7 \\
\hline \multicolumn{3}{|l|}{ Age } \\
\hline $15-24$ years & 187 & 41.2 \\
\hline 25-34 years & 230 & 50.7 \\
\hline$\geq 35$ years & 37 & 8.1 \\
\hline \multicolumn{3}{|l|}{ Family size } \\
\hline $1-2$ & 204 & 44.9 \\
\hline $3-5$ & 201 & 44.3 \\
\hline More than 5 & 49 & 10.8 \\
\hline \multicolumn{3}{|l|}{ Ethnicity } \\
\hline Amhara & 445 & 98.0 \\
\hline Others & 9 & 2.0 \\
\hline \multicolumn{3}{|l|}{ Religion } \\
\hline Orthodox & 402 & 88.5 \\
\hline Muslim & 45 & 9.9 \\
\hline Protestant & 7 & 1.6 \\
\hline \multicolumn{3}{|l|}{ Duration in marriage } \\
\hline $0-5$ years & 212 & 46.7 \\
\hline $6-10$ years & 132 & 29.1 \\
\hline $11-20$ years & 79 & 17.4 \\
\hline above 20 years & 31 & 6.8 \\
\hline \multicolumn{3}{|l|}{ Occupation } \\
\hline House wife & 199 & 43.8 \\
\hline Employee & 199 & 43.8 \\
\hline Students & 23 & 5.1 \\
\hline others & 33 & 7.3 \\
\hline \multicolumn{3}{|l|}{ Monthly income } \\
\hline Up to 500 birr & 50 & 11.0 \\
\hline 501-1000 birr & 129 & 28.4 \\
\hline $1001-2000$ & 131 & 28.9 \\
\hline 2001-3000 & 69 & 15.2 \\
\hline$>3000$ & 75 & 16.5 \\
\hline
\end{tabular}




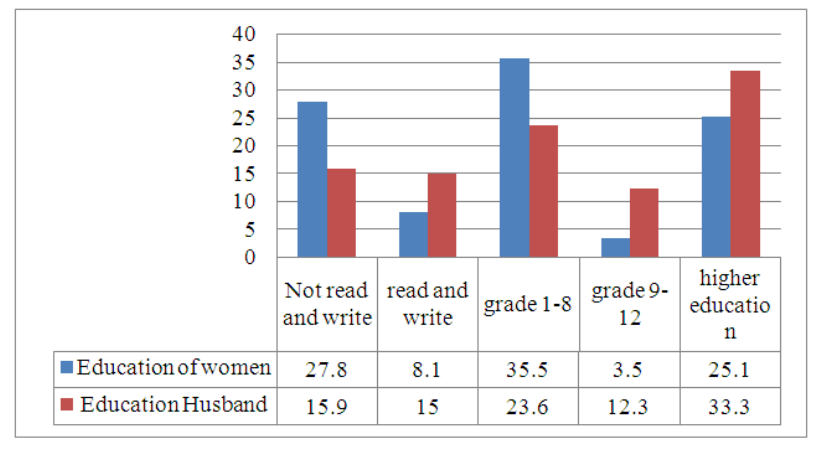

Figure 1. Educational level of women attending ANC and their partners at Felege Hiwot Referral Hospital. July, 2012.

\subsection{Reproductive History of Pregnant Women}

Of the total interviewed women, 432(95.2\%) replied that they knew at least one modern contraceptive method. All the respondents, $454(100 \%)$ knew pills and higher proportion, $426(93.6 \%)$ of them also mentioned injectable contraceptives. On the contrary, only $13(2.9 \%)$ of the participants were aware of vasectomy as family planning technique. Nine in ten, $404(89.0 \%)$, of the respondents also reported that they had ever used contraception sometimes in the past.

Regarding the advantage of contraceptives, majority $(87.7 \%)$ of the women knew function of contraceptives on preventing unwanted pregnancy. However, less than half $(44.5 \%)$ of them knew about post pills and only $1.5 \%$ of the respondents were familiar with the additional advantage of contraceptives to correct irregular menstruation.

Women were asked about the total number of pregnancies and live children they had. Accordingly, half $(50.9 \%)$ of study participants had $2-4$ children and in $41.6 \%$ of the women their most recent pregnancies at a time was their first pregnancy (figure 2).

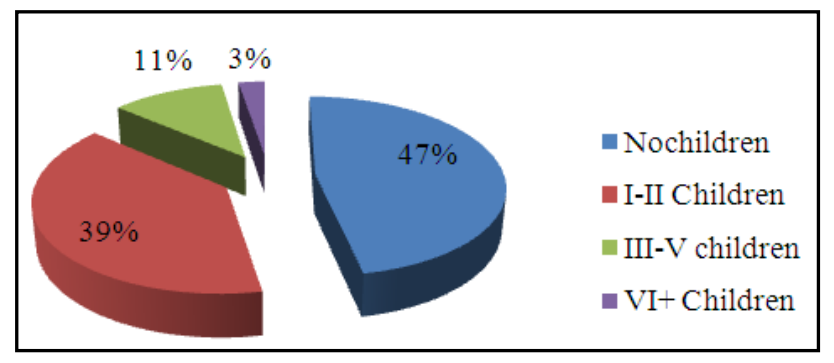

Figure 2. Number of live children among ANC attendees at FHRH, Bahir Dar, July 2012.

Regarding the intentions of women on the current pregnancies $118(26.0 \%)$ of pregnant women reported that their recent pregnancies were unintended. Unintended pregnancy was further split into mistimed and unwanted pregnancies. Of these, $62(13.7 \%)$ of women wanted a child if it had been after two years (those wanting to space child birth-mistimed) and 56(12.3\%) of women reported that they did not want their recent pregnancies at all (unwanted).

Participant women were also asked about their previous history of unintended pregnancy and $65(29.5 \%$ ) of them replied that they had experienced unintended pregnancies other than the recent pregnancy. From those who had unplanned pregnancy, $19(29.2 \%)$ of them mentioned that they had experienced unplanned pregnancy more than once in their life time. Majority (78.5\%) of women who had unplanned pregnancy continued the pregnancy and gave birth. On the other hand, only $2(3.1 \%)$ interrupted the pregnancy and $12(18.5 \%)$ tried to interrupt their pregnancies though they failed.

Of the respondents who had previous history of unintended pregnancy, the most frequent reply given as the reasons for failure to avoid such pregnancies were contraceptive method failure, 20 (30.8\%), and Partner disapproval, 12 (18.5\%). Among those women who became pregnant while using contraceptive (i.e. due to contraceptive method failure) while $13(65 \%)$ of them used according to recommendations, the rest one third did not take the contraceptives given as per the direction given from their health workers (Figure 3).

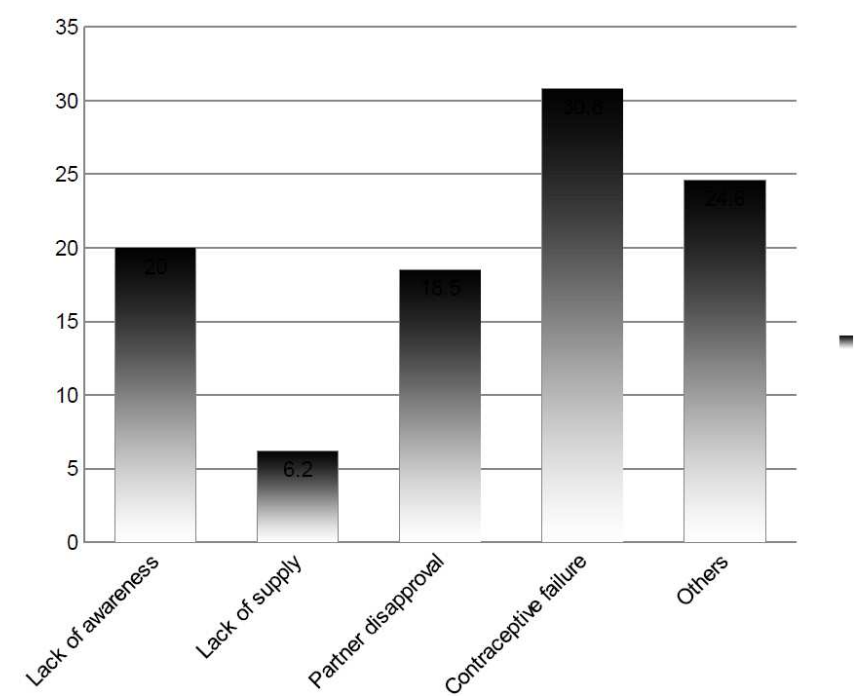

Figure 3. Reasons for unintended pregnancy among women attending ANC clinic in FHRH, Bahir Dar, Ethiopia, July 2012

The finding of this study on the relation between intention of current pregnancy and the number of children revealed that $30(14.0 \%)$ of the pregnancies for women who had not had children were unintended, among whom, 22(10.3\%) were mistimed and $8(3.7 \%)$ were unwanted. Further splitting of this proportion in to the number of live children showed that $33(18.7 \%)$ of the pregnancies were mistimed and $16(9.0 \%)$ were unwanted for women who had 1-2 children. On the other hand, for women who had 3-4 children $58.8 \%$ and for those women who had 5 or more children $75 \%$ of their pregnancies were unintended respectively.

Despite increased trend of unintended pregnancy with number of live children, mistimed and unwanted pregnancies vary in each age category. For women who had no children and for those who had one to two children their mistimed pregnancies were higher than unwanted pregnancies $(10.3 \%$ vs. $3.7 \%$ and $18.7 \%$ vs. $9.0 \%$ 
respectively). On the contrary as the number of children women had increased to 3-4 and 5 and above the proportion of unwanted pregnancies were relatively greater than mistimed pregnancies $(47.1 \%$ vs. $2.9 \%$, and $66.7 \%$ vs. $8.3 \%$ ) respectively (Table 2 ).

Table 2. Intention to current pregnancy versus number of children among pregnant women attending ANC clinic in FHRH, July, 2012(n=454)

\begin{tabular}{|c|c|c|c|c|}
\hline \multirow{3}{*}{ No of children } & \multicolumn{4}{|c|}{ Intention to the current pregnancy } \\
\hline & \multirow{2}{*}{ Wanted pregnancy } & \multicolumn{3}{|c|}{ Unintended pregnancy } \\
\hline & & Mistimed & unwanted & Total Unintended Pregnancy \\
\hline No children & $183(86.0 \%)$ & $22(10.3 \%)$ & $8(3.7 \% \%)$ & $30(14.0 \%)$ \\
\hline 1-2 children & $129(72.5 \%)$ & $33(18.7 \%)$ & $16(9.0 \%)$ & $49(27.5 \%)$ \\
\hline 3-4 children & $21(41.2 \%)$ & $6(2.9 \%)$ & $24(47.1 \%)$ & $30(58.8 \%)$ \\
\hline Five and above & $3(25.0 \%)$ & $1(8.3 \%)$ & $8(66.7 \%)$ & $9(75.0 \%)$ \\
\hline
\end{tabular}

\subsection{Part III. Determinants of Unintended Pregnancy}

According to this study factors that are associated with unintended pregnancy on bivariate analysis at the level of $\mathrm{P}$ value of 0.2 and less were fit in to multivariate logistic regression model. Accordingly, variables such as age of women, education level of women, education level of partner, family size, duration in marriage, wealth status, family planning awareness, the number of pregnancies, live children and previous history of unintended pregnancy were exported in to multivariate logistic regression analysis model.

Using backward stepwise elimination, it was found that educational level of women and family size were remained in the condensed model. The multivariate analysis result of this study declared that educational level of women, family size, partner's awareness on women's utilization of contraceptive before the recent pregnancy and previous history of unintended pregnancy were found to be statistically significant predictors of unintended pregnancy.

Illiterate women (unable to read and write) were three times more likely to experience unintended pregnancy compared to those women educated secondary and above $(\mathrm{AOR}=3.10$, CI: 1.66- 5.78). Likewise, those women who had family size of 3-5 were twice more likely at risk to have unintended pregnancy compared to those who had family size of $1-2(\mathrm{AOR}=2.19 ; \mathrm{CI}: 1.32-3.61)$ and those women who had family size of greater than 5 were nine folds at risk to have unintended pregnancy compared to those who had family size of 1-2 (AOR=8.90; CI: 4.37-18.13).

Previous history of unintended pregnancy showed its association with the current unintended pregnancy. The strongest association was among women who reported previous history of unintended pregnancy $(\mathrm{AOR}=10.6$; $\mathrm{CI}$ : 4.76-23.41) in relative to women with no history of unintended pregnancy. Similarly, awareness of male partners on the utilization of contraceptives of their wives revealed a statistical significant association with unintended pregnancy. Women whose husbands knew contraceptive utilization were almost six times less likely to have unintended pregnancy compared to their counterparts of women whose partners had no awareness on their contraceptive utilization $(\mathrm{AOR}=5.96$; $\mathrm{CI}$ : 1.64- 21.67). According to this study, differences in age of the respondents and intention to current pregnancy, monthly income, duration in marriage, number of children, and number of pregnancy were not associated with unintended pregnancy in the multivariate analysis (Table 3 ).

Table 3. The association between selected socio- economic and demographic variables and intention to recent pregnancies among women attending ANC clinic in FHRH, Bahir Dar, July, $2012(n=454)$

\begin{tabular}{|c|c|c|c|c|}
\hline \multirow{2}{*}{ Variables } & \multicolumn{2}{|c|}{ Intention to current pregnancy } & \multicolumn{2}{|l|}{ OR $(95 \% C I)$} \\
\hline & Unintended & wanted & $\operatorname{COR}(\mathrm{CI})$ & $\operatorname{AOR}(\mathrm{CI})$ \\
\hline \multicolumn{5}{|l|}{ Age } \\
\hline $25-34$ years & $62(27.0 \%)$ & $168(73.0 \%)$ & $1.60(1.003,2.56)$ & $1.10(0.611,1.990)$ \\
\hline 35 and above & $18(48.6 \%)$ & $19(51.4 \%)$ & $4.11(1.96,8.64)$ & $1.01(0.36,2.85)$ \\
\hline \multicolumn{5}{|c|}{ Monthly income ( in ETB) } \\
\hline Up to 1000 & $52(29.1 \%)$ & $127(70.9 \%)$ & $2.38(1.16,4.88)$ & $2.20(0.89,5.45)$ \\
\hline $1001-2000$ & $39(29.8 \%)$ & $92(70.2 \%)$ & $2.47(1.18,8.18)$ & $1.91(0.80,4.57)$ \\
\hline 2001-3000 & $13(18.8 \%)$ & $56(81.2 \%)$ & $1.35(0.56,3.25)$ & $1.35(0.51,3.53)$ \\
\hline$>3000$ & $11(14.7 \%)$ & $64(85.3 \%)$ & 1.00 & 1.00 \\
\hline \multicolumn{5}{|l|}{ Family size } \\
\hline $1-2$ & $28(13.7 \%)$ & $176(86.3 \%)$ & 1.00 & 1.00 \\
\hline $3-5$ & $56(27.9 \%)$ & $145(72.1 \%)$ & $2.43(1.47,4.02)$ & $2.19(1.32,3.61)^{*}$ \\
\hline$>5$ & $31(63.31 \%)$ & $45(36.7 \%)$ & $10.8(5.35,21.90)$ & $8.90(4.37,18.13)^{* *}$ \\
\hline \multicolumn{5}{|c|}{ Duration in marriage } \\
\hline $0-5$ years & $37(17.5 \%)$ & $175(82.5 \%)$ & 1.00 & 1.00 \\
\hline $6-10$ years & $31(23.5 \%)$ & $101(76.5 \%)$ & $1.45(0.85,2.48)$ & $0.97(0.53,1.78)$ \\
\hline $11-20$ years & $30(38.0 \%)$ & $49(62.0 \%)$ & $2.90(1.63,5.15)$ & $1.07(0.53,2.20)$ \\
\hline
\end{tabular}




\begin{tabular}{|c|c|c|c|c|}
\hline \multirow{2}{*}{ Variables } & \multicolumn{2}{|c|}{ Intention to current pregnancy } & \multicolumn{2}{|l|}{ OR $(95 \% \mathrm{CI})$} \\
\hline & Unintended & wanted & COR (CI) & $\operatorname{AOR}(\mathrm{CI})$ \\
\hline Above 20 years & $17(54.8 \%)$ & $14(45.2 \%)$ & $5.74(2.60,12.67)$ & $2.73(1.09,6.87)$ \\
\hline \multicolumn{5}{|l|}{ Educational status of women } \\
\hline Illiterate (unable to read and write) & $48(38.1 \%)$ & $78(61.9 \%)$ & $3.60(1.96,6.58)$ & $3.10(1.66,5.78)^{* *}$ \\
\hline \multicolumn{5}{|l|}{ Primary } \\
\hline Secondary & $48(24.2 \%)$ & $150(75.8 \%)$ & $1.87(1.014,3.36)$ & $1.74(0.96,3.17)$ \\
\hline \&above & $19(14.6 \%)$ & $111(85.4 \%)$ & 1.00 & 1.00 \\
\hline \multicolumn{5}{|l|}{ Partners educational status } \\
\hline Not read and write & $26(36.1 \%)$ & $46(63.9 \%)$ & $2.51(1.39,4.56)$ & $1.09(0.47,2.53)$ \\
\hline Primary education & $51(29.1 \%)$ & $124(70.9 \%)$ & $1.83(1.13,2.96)$ & $0.88(0.46,1.67)$ \\
\hline Secondary and above & $38(18.4 \%)$ & $169(81.6 \%)$ & 1.00 & 1.00 \\
\hline \multicolumn{5}{|l|}{$\mathrm{F} / \mathrm{P}$ awareness } \\
\hline Yes & $105(24.3 \%)$ & $327(75.7 \%)$ & 1.00 & 1.00 \\
\hline No & $10(45.5 \%)$ & $12(54.5 \%)$ & $2.6(1.09,6.18)$ & $3.28(0.15,70.43)$ \\
\hline \multicolumn{5}{|c|}{ Partner knew utilization of $\mathrm{F} / \mathrm{P}$ of women } \\
\hline Yes & $68(20.9 \%)$ & $258(79.1 \%)$ & 1.00 & 1.00 \\
\hline No & $10(41.7)$ & $14(58.3 \%)$ & $2.7(1.15,6.37)$ & $5.96(1.64,21.67)^{*}$ \\
\hline \multicolumn{5}{|l|}{ Number of Px } \\
\hline First px & $26(13.8 \%)$ & $163(86.2 \%)$ & 1.00 & 1.00 \\
\hline II-IV & $67(29.0 \%)$ & $164(71.0 \%)$ & $2.56(1.55,4.23)$ & $1.11(0.19,6.40)$ \\
\hline Five and above & $25(73.5 \%)$ & $9(26.5 \%)$ & $17.42(7.32,41.45)$ & $6.37(0.83,49.25)$ \\
\hline \multicolumn{5}{|l|}{ Number of children } \\
\hline No children & $30(14.0 \%)$ & $188(86.0 \%)$ & 1.00 & 1.00 \\
\hline 1-2children & $49(27.7 \%)$ & $128(72.3 \%)$ & $2.35(1.41,3.90)$ & $2.86(0.28,29.40)$ \\
\hline 3-4 children & $28(54.9 \%)$ & $23(45.1 \%)$ & $7.47(3.81,14.64)$ & $2.84(0.22,36.41)$ \\
\hline Five and above & $8(66.7 \%)$ & $4(33.3 \%)$ & $12.27(3.48,42.28)$ & $3.15(0.10,97.48)$ \\
\hline \multicolumn{5}{|l|}{ Previous hx of unintended pregnancy } \\
\hline Yes & $42(64.6 \%)$ & $23(35.4)$ & $8.75(4.58,16.72)$ & $10.6(4.76,23.41)^{*}$ \\
\hline No & $29(17.3 \%)$ & $139(82.7)$ & 1.00 & 1.00 \\
\hline
\end{tabular}

* Statistically significant at $\mathrm{P}<0.05 \&$ ** statistically significant at $\mathrm{P}<0.001$; OR= Odds Ratio, AOR =Adjusted Odds Ratio; COR=Crude Odds Ratio; $\mathrm{ETB}=$ Ethiopian Birr; $\mathrm{hx}=$ history; $\mathrm{px}=$ pregnancy history

\section{Discussions}

Many women and couples in Ethiopia do not have the knowledge, tools or assistance they need to maintain their reproductive health and have the number of children they desire. Consequently, many women have more children than they want or can care for [7].

In this study, attempt was made to examine the magnitude and predictors of unintended pregnancies based on a sample of 454 women attending prenatal care at Felege Hiwot Referral Hospital. The finding of this study showed that a little more than a quarter, $26 \%$, of women reported their most recent pregnancy as unintended and among all unintended pregnancies, $13.7 \%$ of women wanted a child if it had been after two years and $12.3 \%$ reported that they did not want their recent pregnancies at all. This finding is in line with the Ethiopian Demographic and Health Survey which reported that one fourth of the pregnancies were unplanned [8]which makes the results of this study comparable to the national average. It is also similar to the results from a study in Kersa, East Ethiopia [13]. However, Being the study done in Bahir Dar, Capital of Amhara region and so having physical access to health services, the prevalence of unintended pregnancy is relatively high.
On the other hand, the prevalence of unintended pregnancy in this study was lower than the study done in Harar, East Ethiopia (36.3\%) and Damote Gale district, South Ethiopia, $42.4 \%[14,18]$. Similarly, the finding of the current study was lower than the prevalence reported in Oreland district, Ecuador, 36.3\% and Nepal, 41.2\% [12, 15]. This reduction in this study could be attributed to the progress in the awareness due to the time gap between studies and availability of services in the current study as it was done in the capital of Amhara Region and other factors related to the study areas.

Inadequate knowledge on avoiding unwanted pregnancy, husband disapproval, and method failure were the most frequent reason mentioned by the participants for failure to avoid unintended pregnancies in this study. Majority of the respondents, 95.2\%, were aware of contraceptives. However, this high knowledge of contraceptives appears to contradict with the reported low awareness, $44.5 \%$, on the advantage of post pills and the inadequate knowledge on avoiding unwanted pregnancy as reason cited for the recent unintended pregnancy in $20 \%$ of the respondents.

In every society husbands have pivotal role in the decision of family planning utilization .In this study partner disapproval was the risk factor for unintended pregnancy in 
$18.5 \%$ of study participants 'which calls for initiating activities on male involvement in avoiding unwanted pregnancies. This was higher than the study done in Uganda [23] in which $13 \%$ of pregnancies were unintended due to husband opposition of family planning utilization. The difference could be attributed to cultural and religious differences that are restrictive for Ethiopian women and permissive for Uganda's women in terms of their autonomy in decision making or define their health needs including in the determination of family sizes.

Method failure is a serious problem with great implications both for women as well as for the healthcare facilities providing family planning services: the individual woman could face unwanted pregnancy and its consequences and the system would lose confidence of users and potential users. About $31 \%$ of unintended pregnancies in our study were due to method failure. Although the causes of method failure may need to be investigated in more details, poor counseling during service delivery might be a likely contributing factor.

Educational status of women was one of the predictors of unintended pregnancy in the study area. Illiterate women were three times more likely to experience unintended pregnancy compared to the reference group. This implied that education as having the pervasive impacts on married women's pregnancy intention since it empowers women with knowledge and practice of contraceptive methods leading to reduction of the chance for discontinuity of contraceptive utilization.

It was also noted that family size was predictor for unintended pregnancy in this study. Those women who had family size of 3-5 and greater than 5 were respectively twice, and nine folds, at risk of having unintended pregnancy than the reference groups. This might be an evident that the toll of unmet need for family planning was high due to the fact that these women could already have attained the number of families they desired.

Even though age failed to show significant association with unintended pregnancy in the multivariate logistic regression, the proportion of woman experiencing unintended pregnancy was higher among older women compared to those in the young age groups. The descriptive result showed that proportion of unintended pregnancy increased from $18.7 \%$ among the age groups of $15-25$ years and got pick among the age group of 35 and above with the proportion of $48.6 \%$. The unadjusted OR also showed that unintended pregnancy among the age groups of 25-34 and $35+$ were nearly twice and four folds more likely to be higher than the reference categories of 15-24 years of age. This finding is in line with the Ethiopian DHS 2011[8] and the study done in South Ethiopia [18].This might be explained by the fact that in most instances women at this age have sufficient number of children and they were fatigued due to repeated pregnancies occurred at younger age.

\section{Strength and Weakness}

\subsection{Strength}

The study tried to investigate common but unforeseen problem of maternal morbidity and mortality in the area. It also considered cultural issues through involving the same gender as data collectors as it is common in the community to openly discuss reproductive matters with other women than men.

\subsection{Weakness}

Interviewer administrated questionnaire was used which affect the right to respond freely; still social desirability bias might not be eliminated. The sensitivity of the reproductive issue and expose rationalization (that is reporting a pregnancy that was at first unwanted, later reported as wanted if the pregnancy has continued) of unintended birth might result in under reporting.

\section{Conclusions}

The findings of this study revealed that large proportion of women had unintended pregnancy. The study concludes that no single factor affected unintended pregnancy, rather many factor were interwoven to affect the occurrence of the event. The findings of this study showed that education status of women, family size, and partner awareness on utilization of family planning of their wives, previous history of unintended pregnancy were significant predictors of the recent unintended pregnancy in Felege Hiwot Referral Hospital, North west Ethiopia.

Method failure, more particularly pills, low awareness on contraceptive utilization, and partner disapproval were among the main reasons mentioned by study participants for unintended pregnancy. On the other hand, only few women cited that unintended pregnancies were resulted from low access to family planning techniques. This implies that, efforts to expand access to family planning methods without regard for the provision of effective BCC and quality care might have little benefit in terms of achieving the major objectives of avoiding unintended pregnancy. Based on the key findings discussed above, programs should design to ensure sustainable behavioral changes among community members, strengthening the follow up system on FP workers and beneficiaries, design a strategy to improve inter-spousal communication through peer or informal education and community level orientation. Policy makers, health professionals and health authorities should give due attention to the improvement in the provision of effective IEC, counseling and quality of care. It is very important to have strategies to increase men's participation with their partners in using contraception. Furthermore, undertake further in depth and large-scale study in order to explore underlying causes and design specific interventions. 


\section{Competing Interest}

The authors declare that they have no any competing interests.

\section{Authors' Contribution}

WG: Conceptualized the research problem, designed the study, prepared the proposal, conducted field work, and analyzed the data.

AA: participated in data analysis, the report revision, and manuscript editing

WD: Participated in preparing the manuscript for publication

SL: Revised the proposal and revised the report

\section{Acknowledgements}

We would like to express our grateful heartfelt appreciation to Felege Hiwot Referral Hospital administrators, technical staffs and supervisors of the study for their cooperation and support, giving unreserved time and follow up from the beginning till completion of this research thesis.

Our acknowledgement also extends to study participants and data collectors who were involved in this study.

\section{References}

[1] WHO: Engaging men in changing gender based inequality in health: Evidence from programme intervention, Geneva. 2007.

[2] Amy O, Raegan M, Anne E: Family Planning and the Burden of Unintended Pregnancies. Epidemiol Rev. 2010:32.

[3] Panday, S., Makiwane, M., Ranchod, C., \& Letsoalo, T. Teenage pregnancy in South Africa - with a specific focus on school-going learners. Child, Youth, Family and Social Development, Human Sciences Research Council. Pretoria: Department of Basic Education. August 2009. ISBN No.: 978-0-620-44701-0

[4] Glasier A, Gulmezoglu A, Schmid G, Moreno C, and Van Look P: Sexual and reproductive health: a matter of life and death Lancet. 2006; 368(9547):1595-607.

[5] Amin S, Howden C, Peyman N: A Comparison Study: Risk Factors of Unplanned Pregnancies in a Group of Iranian and New Zealander Women. European Journal of Scientific Research 2009; 26(1):108-21.

[6] Singns S, Wulf B, Hussein R, Bankole A, Sedgh G: Decade of uneven progress, Abortion World Wide. New York: Guttmacher Institute, 2009.

[7] Guttmacher Institute: Benefits of Meeting the contraceptive Needs of Ethiopian Women 2010 series, number 1: 4

[8] CSA: Ethiopian Demographic and Health Survey: Fertility references and Family planning, Addis Ababa Ethiopia 2011:81-108.

[9] Ahmed A: Maternal Mortality Trend in Ethiopia. Ethiop J Health Dev 2010; 24(1):115-22.
[10] Kwast B, KidaneMariam W, EM Said, Fowkes F: Epidemiology of maternal mortality in Addis Ababa: a community-based study. Ethiop Med J 1985; 23:7-16.

[11] Berhan Y, Abdella A: Emergency obstetrics performance with emphasis on operative delivery outcome: Does it reflect the quality of care. Ethiop J Health Dev 2004; 18(2):96-106

[12] Ramesh A, Kusol S, Pramote P: Correlates of unintended pregnancy among currently pregnant married women in Nepal. BMC Int Health Hum Rights 2009;9(17):

[13] Assefa N, Berhane Y, Worku A: Predictors of Unintended Pregnancy in Kersa, Eastern Ethiopia. Reproductive Health 2010; 9(1):1.

[14] Solomon W, Mesganaw F: Unintended pregnancy and induced abortion in a town with accessible family planning services: The case of Harar in eastern Ethiopia. Ethiop J Health Dev 2006; 20(2):79-83.

[15] Isabel G, MiguelSan S: Unintended pregnancy in the Amazon basin of Ecuador: a multilevel analysis. Int J Equity Health 2010; 9(14).

[16] Glei D: Measuring contraceptive use patterns among teenage and adult women. Fam Plann Perspect 1999; 31(2):73-80.

[17] Ramesh A, Kusol S, Pramote P: Demographic, socioeconomic, and cultural factors affecting fertility differentials in Nepal. BMC Int Health Hum Rights 2009; 9(17).

[18] Nigatu R, Tadele K: A population based study on unintended pregnancy among married women in a Gale district in Southern Ethiopia Journal of Geography and Regional Planning. 2011; 4(7):417-27.

[19] AnuManchikanti G: Sexual violence as a predictor of unintended pregnancy, contraceptive use, unmet need, among female youth. Colombia Journal of Women's Health 2011;20(9):1349-56.

[20] Mosfequr R: Women's Autonomy and unintended pregnancy among currently pregnant women in Bangladish. Maternal child health journal 2011:10.

[21] Amaha H, Enqueselassie F: Influence of women's autonomy on couple's contraception use in Jimma town, Ethiopia. Ethiop J Health Dev 2006; 20(3):145-51.

[22] Kamal M, Islam A: Prevalence and socioeconomic correlates of unintented pregnancy among women in rural Bangladesh. Salud Publica Mex 2011; 53(2):108-15.

[23] Shane K, Joy Bradley, Fishel V: Unmet Need and the Demand for Family Planning in Uganda: Further Analysis of the Uganda Demographic and Health Surveys 2008.

[24] Okanlawon K, Reeves M, Agbaje O: Contraceptive use: knowledge, perceptions and attitudes of refugee youths in Oru Refugee Camp, Nigeria. Afr J Reprod Health 2010;14 (4):16-25.

[25] Hubacher D, Mavranezouli I, McGinn E: Unintended pregnancy in sub-Saharan Africa: magnitude of the problem and potential role of contraceptive implants to alleviate it. Contraception Jul 2008; 78(1):73-8.

[26] Gessessew A: Abortion and unwanted pregnancy in Adigrat Zonal Hospital, Tigray, north Ethiopia. Afr J Reprod Health 2010;14(3):183-8. 
[27] Yohannes D: Factors Influencing Women's Intention to Limit Child Bearing in Oromia, Ethiopia. Ethiop J Health Dev 2009;23 (1):29
[28] CSA: Ethiopian Demographic and Health Survey: Fertility references and Family planning, Addis Ababa Ethiopia 2011:81-93. 